\title{
ORBITAL INSTABILITY ZONES OF SPACE BALLOONS
}

\author{
A. V. KRIVOV, L. L. SOKOLOV
}

Astronomical Institute, St. Petersburg University

St. Petersburg, Russia

AND

J. GETINO

Depto. de Matemàtica Aplicada Fund., Facultad de Ciencias Valladolid, Spain

\begin{abstract}
We consider the motion of a spherically-symmetric balloon satellite perturbed by the Earth's oblateness and solar radiation pressure. For equatorial satellite orbits and neglecting the Earth obliquity, the orbit-averaged equations for eccentricity and longitude of pericenter are integrable in quadratures (Krivov and Getino, 1996). The instability zone associated with the saddle separatrix in the phase space has been found and explored in depth. For semimajor axes about two Earth's radii, and for area-to-mass ratios in the order of several tens $\mathrm{cm}^{2} \mathrm{~g}^{-1}$, the amplitude and period of eccentricity oscillations may change nearly twofold under a small change of initial conditions or force parameters. We then restore the actual Earth obliquity of $23^{\circ} .5$ and consider a spatial (non-integrable) problem. Near the saddle separatrix, a stochasticity zone appears that leads to large unpredictable eccentricity variations. The quasirandom motions of space balloons are investigated in terms of two-symbol (0-1) sequences by methods of stochastic celestial mechanics.
\end{abstract}

\section{Introduction}

This paper is a continuation of our study (Krivov and Getino, 1996), dealing with dynamics of spherical Earth satellites with large area-to-mass ratios, moving in high-altitude low-inclination orbits. We take into account two perturbing forces - due to oblateness of the Earth and solar radiation pressure - and make reasonable simplifications. Then, we study the resul-

\section{M. Wytrzyszczak, J. H. Lieske and R. A. Feldman (eds.),} Dynamics and Astrometry of Natural and Artificial Celestial Bodies, 361, 1997.

(C) 1997 Kluwer Academic Publishers. Printed in the Netherlands. 
ting ideal problem and investigate intricate dynamical effects arising from the coupling of the two disturbing forces indicated above.

In Section 2, we consider the planar problem. It means that we artificially set Earth's obliquity to zero and suppose the orbital plane of a satellite to be equatorial. This problem is integrable and admits an exhaustive qualitative analysis to be done. In Section 3, we set the Earth obliquity to its actual value 23.5 and therefore obtain a non-integrable problem. Stochastic features of the motion are investigated here. Section 4 lists our conclusions.

\section{Planar Case: Integrable Problem}

An elliptic orbit in the planar problem is described by three orbital elements: semimajor axis $a$, eccentricity $e$, and longitude of pericenter $\tilde{\omega}$. The semimajor axis $a$ is not subject to secular changes, until the shadowing effects are included, and can be treated as a given parameter. Hence, we should follow the evolution of two elements: $e$ and $\tilde{\omega}$. Instead of $\tilde{\omega}$, it is more convenient to use solar angle, $\phi_{\odot} \equiv \tilde{\omega}-\lambda_{\odot}\left(\lambda_{\odot}\right.$ is the longitude of Sun $)$, which represents the angular distance between the pericenter and direction toward the Sun. As independent variable, we will use $\lambda_{\odot}$ which, since the Earth's orbital eccentricity is neglected, is a linear function of time.

We introduce two dimensionless parameters characterizing the strength of the perturbing forces: radiative parameter $C$ and oblateness parameter $W$. The first of them is defined as

$$
C \equiv \frac{3}{2} \sigma \frac{n}{n_{\odot}}, \quad \sigma \equiv \frac{F_{p r}}{F_{g r}}=\frac{F_{\odot} a^{2}}{G M c} \gamma,
$$

where $n$ and $n_{\odot}$ are mean motions of the satellite and Sun, $\sigma$ is the ratio of radiative to gravity force, $F_{\odot}$ is the solar flux at $1 \mathrm{AU}, G M$ is the Earth's gravitational parameter, $c$ is the speed of light, and $\gamma$ is the area-to-mass ratio of the satellite. We assume the reflectance factor to be unity.

The oblateness parameter $W$ is introduced as

$$
W \equiv \frac{3}{2} J_{2}\left(\frac{R}{a}\right)^{2} \frac{n}{n_{\odot}},
$$

where $J_{2}$ and $R$ denote the oblateness coefficient and equatorial radius of the Earth, respectively. The product $n_{\odot} W$ equals the precession rate of $\tilde{\omega}$ due to $J_{2}$ for a nearly circular orbit. Numerically, $W=1$ at $a=1.94 R$.

The orbit-averaged equations for $e$ and $\phi_{\odot}$ can be written in a semicanonical form (Krivov et al. , 1996; Hamilton and Krivov, 1996):

$$
\frac{d e}{d \lambda_{\odot}}=-\frac{\sqrt{1-e^{2}}}{e} \frac{\partial \mathcal{H}}{\partial \phi_{\odot}}, \quad \frac{d \phi_{\odot}}{d \lambda_{\odot}}=\frac{\sqrt{1-e^{2}}}{e} \frac{\partial \mathcal{H}}{\partial e}
$$


with the autonomous "Hamiltonian"

$$
\mathcal{H}=\sqrt{1-e^{2}}+C e \cos \phi_{\odot}+(W / 3)\left(1-e^{2}\right)^{-3 / 2}
$$

and therefore admit an integral of the motion

$$
\mathcal{H}\left(e, \phi_{\odot}\right)=\text { const. }
$$

Writing the equations of motion in semicanonical form (1)-(2) allows one to perform an exhaustive analysis of their solutions for various combinations of the force parameters. This analysis has been done in (Hamilton and Krivov, 1996) and (Krivov and Getino, 1996). It turns out that the solutions possess some peculiar properties, including drastic changes in the dynamics under small changes of the force parameters or initial data.

Here, we only give an illustrative example. In Figures 1a,b,c, we show the phase portraits of the dynamical system for the semimajor axis $a=2.5 R$ that corresponds to the oblateness parameter $W=0.409$. The radiative parameter $C$ equals to $0.100,0.122 \ldots$, and 0.150 , respectively, that corresponds to $\gamma=146,179$, and $220 \mathrm{~cm}^{2} \mathrm{~g}^{-1}$. We show only one half of a portrait for $\phi_{\odot} \in\left[0,180^{\circ}\right]$ because of reflection symmetry $\phi_{\odot} \rightarrow 360^{\circ}-\phi_{\odot}$. In all cases, the phase portrait $\left(e, \phi_{\odot}\right)$ contains five fixed points: saddles $P_{1}=\left(0,90^{\circ}\right)$ and $P_{2}=\left(0,270^{\circ}\right)$, a maximum $P_{3}=\left(e_{3}, 0\right)$, a saddle $P_{4}=\left(e_{4}, 0\right)$, and a minimum $P_{5}=\left(e_{5}, 180^{\circ}\right)$. There exists a saddle separatrix, a pair of phase curves that start from $P_{4}$ and end at the same point. Another phase curve of special interest is the $e=0$ trajectory associated with an initially circular orbit. The $e=0$ trajectory always starts from the point $\left(e=0, \phi_{\odot}=90^{\circ}\right)$ and may lie below the saddle separatrix, coincide with it, or go above the separatrix, as illustrated by Figures 1a,b,c, respectively. In the first case, the maximum eccentricity of $e_{\max }=0.37$ is reached at $\phi_{\odot}=0$, whereas in the third case $e_{\max }=0.81$ is reached at $\phi_{\odot}=180^{\circ}$. Accordingly, a striking jump in the eccentricity occurs under a small change in the radiative parameter, namely when $C$ passes through the value 0.122 (the second case).

Another way to see the sharp changes in the dynamics is to vary the initial data. If we start two trajectories from two points in the phase space, which are close enough, but lie on the different sides of the separatrix, we have drastically different solutions. This is illustrated by Figures 1d,e,f. These panels are drawn for the same values of $C$ as Figures 1a,b,c, respectively, and portray the eccentricity histories for the pairs of trajectories, lying close to the separatrix on the opposite sides of it. In Figure 1d, the initial data are $\left(e=0.08, \phi_{\odot}=180^{\circ}\right)$ and $\left(e=0.10, \phi_{\odot}=180^{\circ}\right)$; in Figure 1e, they are $\left(e=0.01, \phi_{\odot}=80^{\circ}\right)$ and $\left(e=0.01, \phi_{\odot}=100^{\circ}\right)$; in Figure 1d, we chose $\left(e=0.12, \phi_{\odot}=0\right)$ and $\left(e=0.14, \phi_{\odot}=0\right)$. Both amplitude and period of eccentricity oscillations differ nearly twofold! 

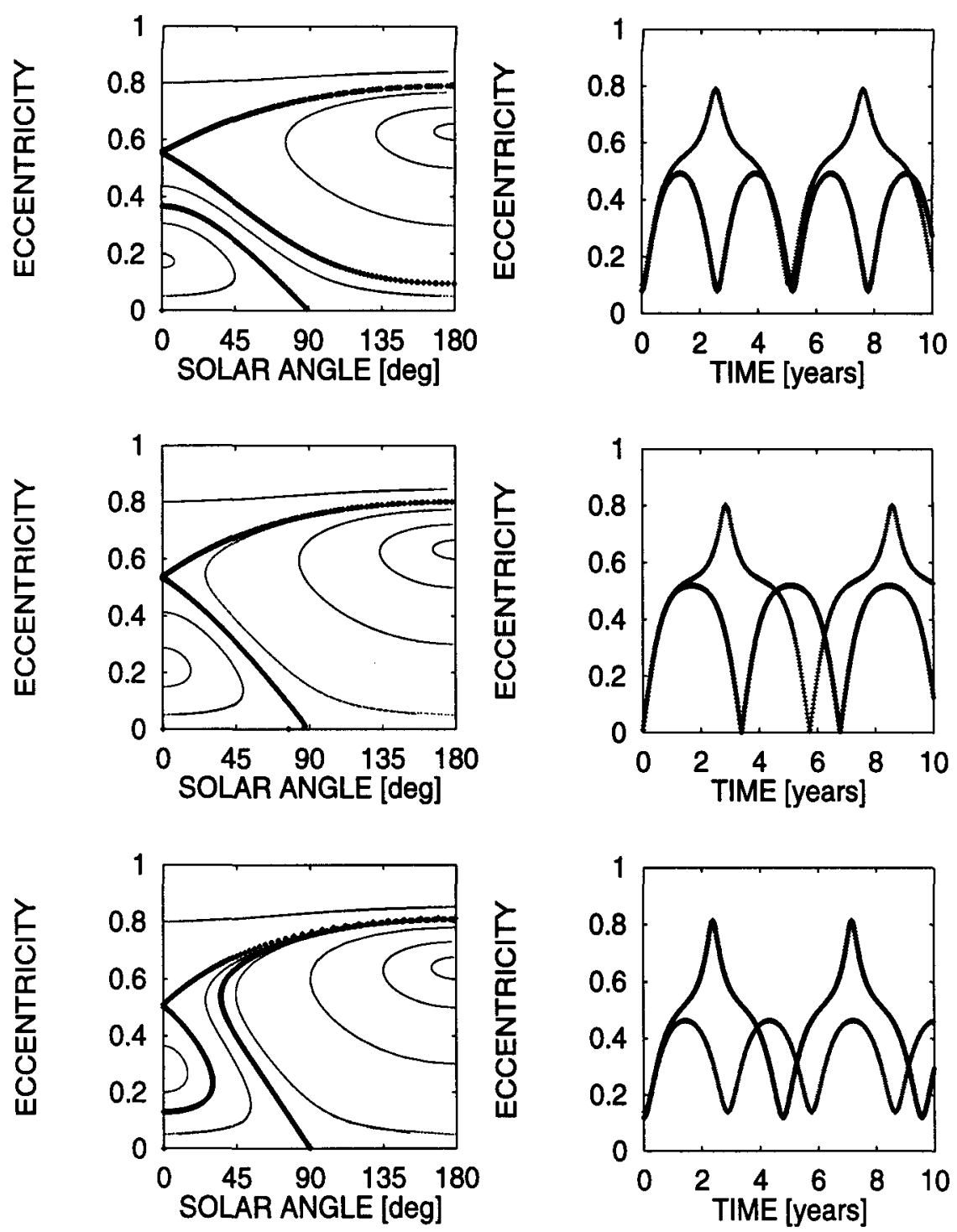

Figure 1. The phase portraits (left panels) and eccentricity histories (right ones) for Eqs. (1) for several special choices of force parameters and initial data. See text for further explanation.

\section{Spatial Case: Non-Integrable Problem}

Now, we come to a more general case. We take into account the tilt of the Earth's equatorial plane to the ecliptic. Besides, we allow an initially zero orbital inclination (with respect to equatorial plane) to evolve, as it must, since the radiation pressure force no longer acts in the equatorial 
TABLE 1. Binary-coded trajectories within the stochasticity zone.

\begin{tabular}{|c|c|c|}
\hline $\mathbf{a}$ & $\mathbf{b}$ & c \\
\hline oOooOooooo & oOoooooooo & oOoooOoOoO \\
\hline ○OoOooO॰Oo & ○OoooOooOo & 0O00000000 \\
\hline oOoOoOoOoO & oOoooOoOo & oOoooOoOoO \\
\hline oOoOoOoOoOo & oOoooOoOoO & ○Ooooooooo \\
\hline oOoOoOıOıOo & oOoooooooo & ○OoooOoOoO \\
\hline ○OoOoOoOoOoO & ○OooooOoOo & ○Oo0000000 \\
\hline oOoOoOoOoOoO & oOoooooooo & ¡Oloooooooo \\
\hline oOoOoOoOoOoO & oOooooOoo & ○Ooooooooo \\
\hline ○OoOoOoOoOoO & ○OoooOoooo & oOoooooOoO \\
\hline oOoOoOoOoOoO & ○OooOoOoO & ○OoooooooO \\
\hline oOoOoOoOoOoO & oOooOooOoO & ○OoooOoOoO \\
\hline
\end{tabular}

plane. As a method of investigation of the non-integrable problem, we use numerical integrations of the orbit-averaged equations of motion derived in (Krivov et al., 1996, Eqs. (17)-(20)). A question to ask is: what properties of the solutions of the planar (integrable) problem hold true and what new properties appear?

A general theory of dynamical systems suggests that the solutions are likely not to change appreciably, as long as they lie far enough from the saddle separatrix. On the contrary, the behavior is quite different near the separatrix where a stochasticity zone should appear. To describe the motions in this zone, we can use the methods of symbolic dynamics. Each trajectory is coded by an infinitely long sequence of binary symbols. We use 0 and $O$ to mark that a trajectory passes along the lower and upper branches of the separatrix, respectively. In other words, a symbol "o" appears when eccentricity reaches its minimum (becomes $\leq 0.2$ in our numerical examples), and a symbol " $\mathrm{O}$ " is added into the sequence when $e$ attains a maximum (becomes $\geq 0.4$ ). After a long "stay" near the saddle point $P_{4}$, a trajectory leaves it along one of the two branches of the separatrix, either lower ("o") or upper ("O") ones, with the next symbol being not determined by a preceding one.

The following numerical example illustrates stochastic motions near the saddle separatrix. Consider a family of trajectories parametrized by an initial value of solar longitude $\lambda_{\odot}$. The other initial data and parameter values are: $C=0.01$ and $W=0.8$ (or $a=2.05 R=13000 \mathrm{~km}$ and $\gamma=16 \mathrm{~cm}^{2} \mathrm{~g}^{-1}$ ); Earth's obliquity $\varepsilon=23.5$; initial eccentricity $e_{0}=0.12511536$; initial inclination $i_{0}=0$; initial solar angle $\phi_{\odot}=180^{\circ}$. Table 1 presents three series of 
binary-coded trajectories. Each of them contains 11 trajectories with different initial values of solar longitude: $\lambda_{\odot}$ from 0.0 to $1: 0$ with step 0.1 (case "a"); $\lambda_{\odot}$ from 0.0015 to 0.0016 with step $10^{-5}$ ("b"); $\lambda_{\odot}$ from 0.00154314 to 0.00154315 with step $10^{-9}$ ("c"). These three sequences of $\lambda_{\odot}$ are thus nested, with the next one being much narrower than the previous one. The left column shows that the width of the stochasticity zone is $\sim 1^{\circ}$; beyond this zone the trajectories have one and the same code. Conversely, within the zone an arbitrarily small change in initial $\lambda_{\odot}$ causes an unpredictable change in the trajectory code! It resembles a fractal structure.

\section{Conclusions}

1. We considered a new integrable case of the photogravitational planetocentric problem with two perturbing forces - oblateness of a planet and solar radiation pressure - and applied the results to space balloons that orbit the Earth. Integrability of the problem allows one to fully describe all variants of the orbital evolution of a balloon satellite in the planar approximation (i.e. neglecting the Earth's obliquity and for equatorial orbits). We particularly found the regions in the phase space where small changes in the force parameters or initial data may cause sharp orbital changes.

2 . We then restored the actual Earth's obliquity of 23.5 and considered a non-integrable spatial problem, but yet confined ourselves to initially equatorial satellite orbits, so that the problem was close to an integrable one. Numerical investigations of this "perturbed" system show that the trajectories in the spatial case are similar to those in the planar problem. The exception is for vicinities of the saddle separatrix in the phase space, where a stochastic zone arises. In this zone, the eccentricity oscillations are large and practically unpredictable, so that the satellite orbits are very unstable.

Acknowledgements. A. Krivov and L. Sokolov were supported by the RBRF grant No. 96-02-19638. J. Getino has been partially supported by CICYT, Project No. ESP93-741.

\section{References}

Hamilton, D.P. and Krivov, A.V.: 1996, "Circumplanetary dust dynamics: Effects of solar gravity, radiation pressure, planetary oblateness, and electromagnetism", Icarus 123, 503-523.

Krivov, A.V. and Getino, J.: 1996, "Orbital evolution of high-altitude balloon satellites", Astron. Astrophys., in press.

Krivov, A.V., Sokolov L.L., and Dikarev, V.V.: 1996, "Dynamics of Mars-orbiting dust: Effects of light pressure and planetary oblateness", Celest. Mech. \& Dyn. Astron.63, 313-339. 